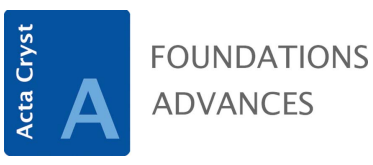

ISSN 2053-2733

\section{Quantum Field Theory and Condensed Matter. An Introduction. By Ramamurti Shankar. Cambridge University Press, 2017. Pp. 450. Price GBP 59.99 (hardback). ISBN 9780521592109.}

\author{
Dragi Karevski*
}

Université de Lorraine, CNRS, Laboratoire de Physique et Chimie Théoriques, Nancy, France. *Correspondence e-mail: dragi.karevski@univ-lorraine.fr

Keywords: book review; quantum field theory

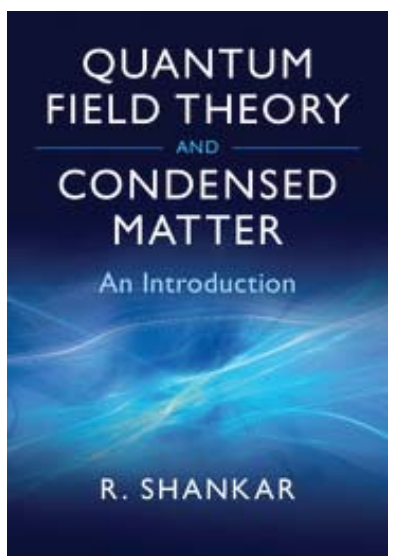

(C) 2018 International Union of Crystallography
This short introduction to quantum field theory techniques used to tackle key topics of modern condensed matter physics is mostly intended for the diligent graduate student but experienced researchers may also find it a valuable resource. It provides a broad review of many different techniques and models used daily in the theoretical condensed matter community. The presentation is done in a quite individual and elegant way; however, in some places this may be at the cost of losing the inexperienced reader who might be confused by the rather sophisticated arguments.

The book starts with a brief introduction to standard thermodynamics and statistical mechanics presented $a$ la Callen. The following five chapters provide a survey of the basic concepts of the Ising model, transfer matrices, the general correspondence between statistical and quantum mechanics and the concept of path integrals, with a full chapter devoted to coherent state path integrals for spins, bosons and fermions. This part of the book can be used self-consistently as a source for an advanced statistical mechanics course at the master degree level.

After that, in the remaining chapters of the book, Shankar covers a wide variety of models ranging from the celebrated classical two-dimensional Ising model, whose original Onsager's exact solution is presented here along the lines drawn by Lieb, Schultz and Mattis, to the $X Y$ model and $Z_{q}$ gauge theories, and finally to models developed for the quantum Hall effect such as the Bohm-Pines or Chern-Simons theories.

In the middle of the book, there are six chapters giving an extensive survey on the renormalization group theory (a book within a book, as Daniel Arovas wrote) and two self-contained chapters dealing with bosonization. Again, here, these chapters may be used self-consistently in order to teach the material.

All in all, even if this book is definitely not a self-sufficient introduction to quantum field theory techniques nor to the most recent topics of condensed matter physics, it is nevertheless a very good way of approaching these topics. 